\title{
At crossroads between laboratory disciplines and medical advancements - The Center for Molecular Medicine at the Karolinska University Hospital
}

\author{
Lars Terenius
}

Published online: 25 March 2009

(C) Springer-Verlag 2009

\begin{abstract}
The Center for Molecular Medicine (CMM) was conceived and built to respond to the challenges presented by the still common chronic diseases such as atherosclerosis, rheumatoid arthritis, diabetes, allergy, and alcoholism. The Karolinska University Hospital has a proud history of research with developments such as the pacemaker and the gamma-knife. The nearby Karolinska Institutet has a strong presence internationally on the basic sciences. However, the challenges of the "new biology" and the access to the complete human genome, transcriptome, and proteome raised the need for a new research institute that could meet the experimental requirements for translational research. A Foundation was established in 1994 with the goal to build and govern the new enterprise. After an intense fundraising campaign, building could start and CMM (Fig. 1) was inaugurated in 1997. Through more than 10 years of existence, it has evolved into a multidisciplinary research institute with research in four programs, Cardiovascular and Metabolic Diseases, Infection and Immunity, Neuropsychiatric Diseases, and Medical Genetics. Performance parameters have been introduced and scientific impact and relevance are followed annually. Transparency and collaboration between groups (now 28 groups with an approximate total of 400 people engaged in research) and leadership training for junior faculty are means to stimulate "centerness".
\end{abstract}

L. Terenius $(\bowtie)$

The Center for Molecular Medicine,

Karolinska University Hospital,

L8, Stockholm 171 76, Sweden

e-mail: Lars.Terenius@ki.se
The Karolinska University Hospital is located on the northern outskirts of Stockholm close to the Karolinska Institutet. The hospital was built approximately 70 years ago and officially inaugurated in 1940. It has a modular layout with separate buildings for different clinical specialties. Research was given priority in the sense that laboratories were located in proximity to and run by each clinic. This arrangement was instrumental in bringing researchers close to clinical realities, but hardly optimal in terms of efficient use of research resources.

The great advances in molecular and cellular biology in the 1990 s, culminating with the complete sequence of the human genome in 2000 , became an impetus for the idea that competitive laboratory research required resources on another scale in terms of equipment, experimental knowhow, and a common knowledge base. The idea of one building accommodating many of the research groups spread out in different existing buildings, also allowing an attractive environment for new recruitments, took shape. A new building, rather than upgrading existing laboratory space, required economic resources that were not available within the hospital budget at the time. Thus, a task force was set up with the goals to find a suitable location for a new building and provide an outline of physical requirements for a building that would fulfill the purpose to provide a platform for the "new biology". Also important was the translation to clinical practice, and to ascertain costs for such a pursuit. Since it was obvious that funds had to be raised, at least in part from donations, the CMM Foundation was instituted, with the purpose to raise financial support. In the negotiations with the Hospital and the Stockholm County, conditions were worked out and a suitable location for the new CMM building was identified (Fig. 1). The building would be localized in close vicinity to the main building of the hospital and 


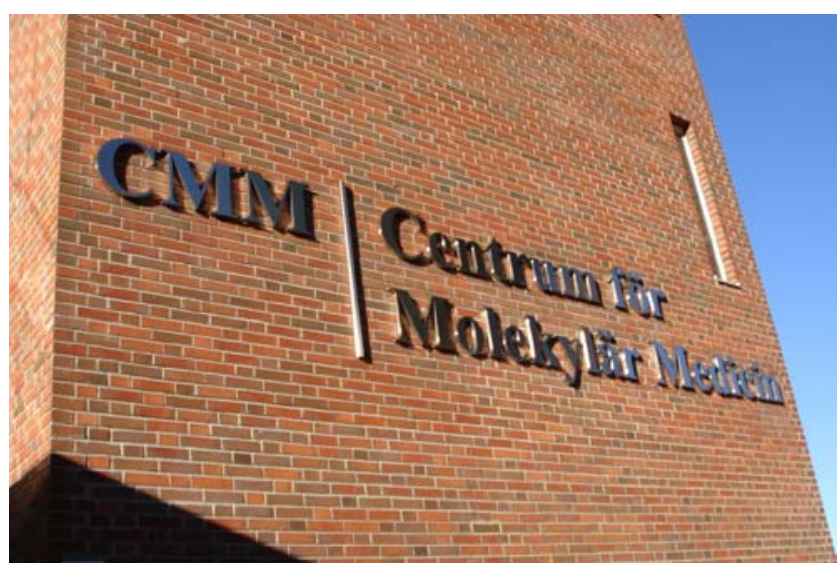

Fig. 1 The Center for Molecular Medicine entrance sign

linked to two older buildings, both with research areas. Thus, besides being the desired new platform for laboratory research, CMM would also be easily accessible from the clinics and partner with other research activities in nearby buildings. This localization seemed ideal for the purpose.

In creating a foundation, CMM acquired an independent status. It would not be under the guidance of The County Council, otherwise responsible for surrounding hospital buildings. On the other hand, the hospital agreed to enter a long-term lease on the building. The crucial question became the funds for construction of the building. In an agreement with the County Council, the CMM Foundation would raise at least $50 \%$ of the total capital by donations and cover remaining costs with a bank loan. On these grounds, the CMM Foundation under the auspices of the Stockholm County Council was founded in 1995. The establishment of this Foundation and the start of the project did not go smoothly; there were voices raised against this initiative as it would create a more complex situation at the hospital in terms of governance with a new independent operator in charge of a building for research.

Fundraising was, by ardent work, successful and by November 1996 the cornerstone was laid. Donations were more generous than the stipulated minimum, as over $60 \%$ of the total cost of the building became available. After a swift planning and building period, the first laboratories were occupied and running in February 1997. The CMM building was officially opened on May 22, 1997.

The CMM building is quite unique in that its concept and design was entirely based on scientific needs. The design is open with no strict boundaries between different laboratories and with space for common activities such as technology platforms etc. The construction lends itself to change, walls can be pulled down or raised, and working spaces easily altered with a minimum of cost. The signum of the building is openness, with large windows on all outer walls (Fig. 2) and inner glass walls lining both sides of the center corridor with common facilities and shared offices. This design has proven practical and inviting for interaction and joint projects.

\section{The CMM mission}

The explicit purpose of CMM is research in common, chronic diseases. In the statutes are mentioned cardiovascular disease, rheumatoid arthritis, metabolic diseases, neuropsychiatric diseases, and allergy. It is recognized that chronic disease may have its roots in genetic disposition. Therefore, it became important to have a strong emphasis on medical genetics, which as a discipline was added to the statutes. Another way to look at the common chronic diseases is that they affect many and are therapeutic failures. Besides genetic disposition, infections and other environmental conditions including early life experience and lifestyle are contributing factors. The complex interplay between these factors makes it hard to establish causal links. Large cohorts of patients may be required to obtain conclusive evidence for causality. The advanced level of information that can be obtained in various registries in Sweden is a unique asset that has been used extensively in research at CMM. The multitude of diagnoses represented at CMM allows comparative studies on a broad range of diseases which are seemingly different, but which may have a common root.

Research on such common denominators has been encouraged at CMM and a special call for grant proposal was awarded support, although modest, from the CMM Foundation. A major theme at CMM is translational research which has been strongly encouraged since CMM

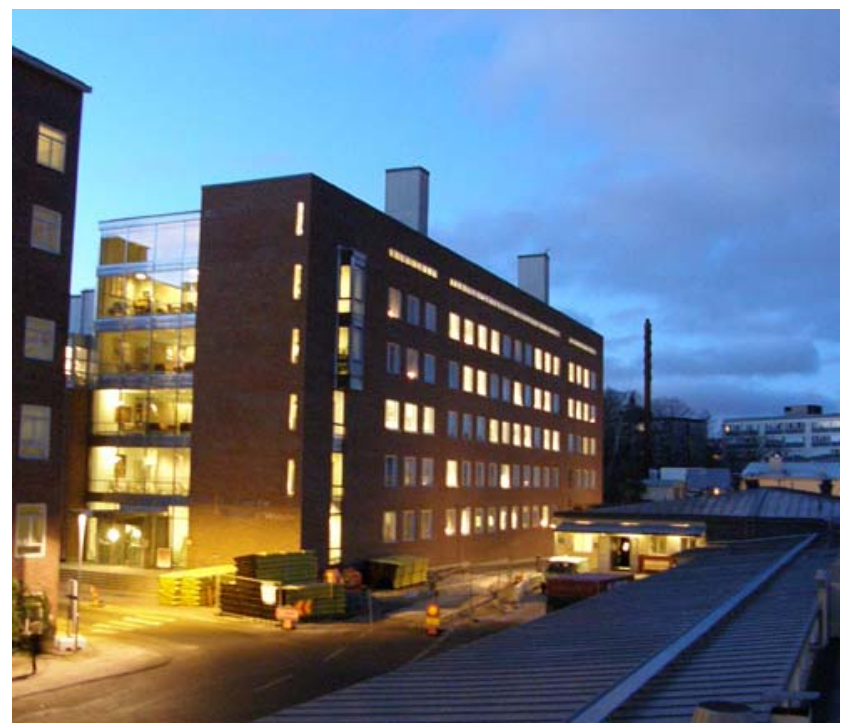

Fig. 2 Late hours at CMM 
was incipient. To encourage the visibility of CMM in the clinic and to stimulate research at CMM, the Foundation has attracted funding from granting agencies to allow physicians with a $\mathrm{PhD}$ in a laboratory discipline to return to laboratory science on a $50 \%$ basis, maintaining clinical work on the remaining $50 \%$ of their time. These fellowships run for 2 years and can be renewed for another 2 years. This program has been very successful and recruited clinicians to do laboratory work. So far, 18 fellowships have been awarded and for every fellowship there have been 2- to 3-fold more applicants.

CMM was originally built for an estimated number of 250 people working in the labs. As of 2008, this has been outnumbered and at the moment over 400 individuals work in the building at least part time.

\section{The CMM model}

Strategic recruitments at the senior scientist level have been facilitated by the opportunity to join CMM. These recruitments were all outside of the Stockholm area and together with already existing research groups at the hospital formed the basis for scientific development. Movement forward by aligning and coordination was essentially ad hoc and served to identify scientific strongholds. Interaction and collaboration was encouraged and CMM started to act as an independent body raising financial support for common activities such as technology platforms. An international scientific advisory board was appointed at an early stage with the explicit role to evaluate scientific output as well as "centerness", i.e., to what extent there would be a common and shared knowledge base and common projects. In the process of giving CMM identity, the concept "intellectual capital" was introduced on the advice from the Steering Board of the Foundation. The intellectual capital, defined as relational capital, structural capital, and human capital, gives an index of what CMM is "when everybody has left the building". Outcome of the research in the short term is analyzed by bibliometric analysis; mid-term results would be clinical trials, new medical treatments, or diagnostic programs. Long-term results and impact for health (preventive medicine) and healing are communicated in various ways including interviews, debates, or seminars. Research at $\mathrm{CMM}$ has reached the public eye on many occasions.

A few years ago, CMM took the initiative to offer junior faculty the opportunity to participate in a course in scientific leadership. The program is run at CMM over one academic year, each with ten participants who meet individually with a professional (external) coach and in group sessions, frequently with an invited guest from the outside world, organizations, companies, etc. The program has become much appreciated and not only promotes the performance of the individual but also promotes interactions between research groups (no group can have more than one participant each year).

\section{CMM and the world outside}

There are ample procedures to measure scientific excellence. CMM uses bibliometric analysis and in particular citation impact. Comparison with other national and international universities gives a favorable result (the interested reader may consult http://www.cmm.ki.se/). It is more difficult to measure relevance. We have begun to use network analysis to identify interactions between groups at $\mathrm{CMM}$ and interactions between CMM and the clinics. The analysis shows that CMM is very well connected with virtually every clinic at the academic hospitals. A similar analysis on connections within Sweden and international connections with the EU countries and USA also point to a very substantial network of collaboration and common projects.

\section{"Turning the world upside down"}

As you enter the CMM building, you face an art object by world-renown artist, Anish Kapoor. The object, a concave mirror in stainless steel $2 \mathrm{~m}$ in diameter, is incorporated in the wall (Fig. 3). As you approach, your own body grows

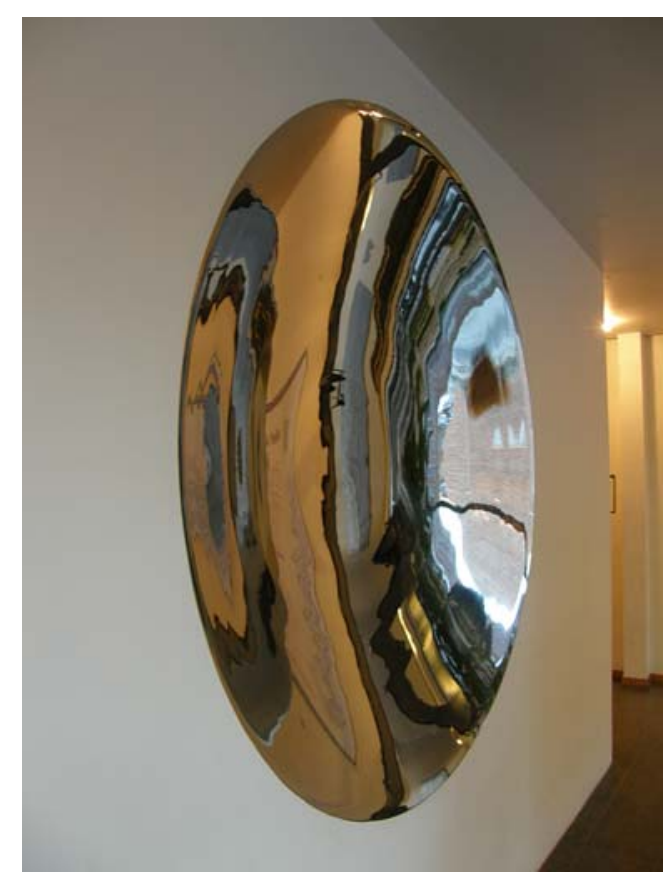

Fig. 3 "Turning the World Upside Down IV" 
and the outside world ceases to exist. Even if collaboration and research groups make collective efforts, it is still the creativity of the individual that is pivotal. The scientific inquiry and the curiosity of the human mind remain key to success.

\section{CMM present and future}

CMM was created in response to the need for advanced research opportunity at the bench level enforced by the intimate contacts with The Karolinska Institutet and close physical and intellectual proximity to the Karolinska University Hospital. Current studies are exemplified in the appended articles of this journal's issue. The flexible design of the building has adapted well to the increase in staff and the expanding activities during the first more than 10 years. This demonstrates that $\mathrm{CMM}$ has been successful in establishing high caliber research. The CMM Foundation guarantees certain independence and at the same time oversees the overall goal to facilitate research in the common chronic diseases. 